\title{
On the discrepancy between the objective and risk neutral densities in the pricing of European options
}

\author{
IJH Visagie* $\quad$ GL Grobler ${ }^{\dagger}$
}

Received: 11 April 2019; Revised: 14 June 2019; Accepted: 14 June 2019

\begin{abstract}
A technique known as calibration is often used when a given option pricing model is fitted to observed financial data. This entails choosing the parameters of the model so as to minimise some discrepancy measure between the observed option prices and the prices calculated under the model in question. This procedure does not take the historical values of the underlying asset into account. In this paper, the density function of the log-returns obtained using the calibration procedure is compared to a density estimate of the observed historical log-returns. Three models within the class of geometric Lévy process models are fitted to observed data; the Black-Scholes model as well as the geometric normal inverse Gaussian and Meixner process models. The numerical results obtained show a surprisingly large discrepancy between the resulting densities when using the latter two models. An adaptation of the calibration methodology is also proposed based on both option price data and the observed historical log-returns of the underlying asset. The implementation of this methodology limits the discrepancy between the densities in question.
\end{abstract}

Key words: Pricing, optimisation, financial markets, stochastic processes.

\section{Introduction}

It is well known that the arbitrage free price of an option can be calculated as the discounted value of the expected payoff of the option in question. However, this expectation is not taken with respect to the probability measure underlying the market (referred to as the objective probability measure), rather this expectation is calculated with respect to

${ }^{*}$ Department of Statistics, University of Pretoria, South Africa, email: jaco.visagie@up.ac.za

${ }^{\dagger}$ Department of Statistics, North-West University, South Africa, email: gerrit.grobler@nwu.ac.za 
some other probability measure (referred to as the risk neutral measure). This paper numerically investigates the discrepancy between these measures. Specifically, by comparing the densities associated with the two probability measures it is demonstrated that there is often a substantial discrepancy between these densities.

The efficient market hypothesis states that all of the information relevant to the prices of options is contained in the observed market price of these options; see, for example, [9]. This serves as an explanation as to why there is a difference between the objective and risk neutral measures. The former can be estimated from historical stock price data, while the latter is influenced by information which only become available after the historical stock prices have been realised. However, there is a close relationship between these two measures. If the difference between the measures is substantial, then the option prices resulting from the model considered are calculated under the assumption that the future behaviour of the stock price will be considerably different from its historical behaviour.

In practice, option pricing models are often fitted using data from European options. The resulting model is then used in order to calculate the prices of exotic options. Large differences between the estimated density of the observed log-returns and the density used for the calculation of option pricing seems especially disconcerting in this case.

Ross, in [14], formulates the recovery theorem. The techniques developed in the mentioned paper are concerned with the link between the density function used for the pricing of financial derivatives and the density of the log-returns of the underlying asset. These techniques have applications in quantifying the level of risk aversion present in a given market. Note that the approach used in the current paper is quite different from the approach used by Ross. In the current paper, the difference between the two densities mentioned is compared. The aim of this paper is to demonstrate the substantial differences between the density of the log-returns of the underlying asset and the risk neutral density.

When fitting a given financial model to observed option prices, a method known as calibration is often employed. Calibrating a model to a set of observed option prices entails choosing the parameters of the model so that the option prices calculated using the model correspond as closely as possible to the observed option prices. This procedure will be made precise below.

In this paper, three geometric Lévy process models are considered; the first is the celebrated Black-Scholes model, while the remaining two are the geometric $N \circ I G$ and Meixner models respectively. The Black-Scholes model, which is based on the geometric Brownian motion, contains two parameters, while the remaining two models contain four. When calibration is used in order to fit these models to observed option prices, it is observed that the risk neutral measure closely resembles the objective measure in the case of the Black-Scholes model. However, it will be shown that, when one of the other models is used, the density of the obtained risk neutral measure can be markedly more leptokurtic than is the case for the density associated with the objective probability measure.

It is common practice to report the parameters of the models used in terms based on annual log-returns. However, estimating the parameters of a financial model based on annual data is not feasible for two reasons. Firstly, in this case, there is often simply not enough historical data available in order to estimate parameters with acceptably small 
standard errors. Secondly, even if a sufficient amount of historical data were available, the prices of options should be calculated under current market conditions. This means that returns observed several years before are outdated and should not be taken into account when fitting the model. As a result, the models used in practice are often fitted using daily data and the parameters reported are shown in annual terms. This mismatch in the time horisons considered can obscure some of the differences between the objective and risk neutral measures; this is demonstrated using a numerical example in the paper.

The remainder of the paper is structured as follows. In Section 2 a brief overview of option pricing is outlined. In Section 2.1 the theory underlying arbitrage free option pricing, with an emphasis on the theoretical relationship between the objective probability measure and the risk neutral measure, is provided. Section 2.2 shows how a risk neutral measure can be obtained from an objective measure by using a change of measure technique called the Esscher transform. This technique requires that the objective measure be estimated using historical price data of the underlying asset. The calculation of option prices is discussed in Section 2.3. In Section 2.4, a calibration procedure often used in order to obtain a risk neutral measure is discussed. This risk neutral measure is obtained by only taking observed option price data into account.

In Section 3, the three models used in this paper are discussed. Each of these models are from the class of geometric Lévy models. In Section 3.1 the Black-Scholes model is discussed. The main advantage of this model is the ease with which option prices can be calculated. However, the inability of this simplistic model to accurately represent the leptokurtic and skew nature of the distributions of observed financial log-returns is well documented; see, for example, [7]. As a result, attention is turned to the more flexible geometric $N \circ I G$ (Section 3.2) and Meixner (Section 3.3) models.

In Section 4 the numerical results pertaining to the pricing of options in observed financial markets are presented. For each of the models under consideration, various risk neutral measures obtained in different ways are considered. The presented analyses focus on the accuracy with which the various risk neutral measures are able to replicate the observed option prices, as well as the discrepancy between the density associated with the risk neutral measure and the density of the estimated objective measure. Section 5 presents the main conclusions of this empirical study.

\section{Option pricing in theory and practice}

To a large extent, there exists a disconnect between the option pricing theory developed in an abstract mathematical framework and the practical implementation of these theoretical concepts. Below, in Section 2.1, the mathematical theory underlying the calculation of arbitrage free option prices are considered, including the estimation of the distribution of the log-returns of the stock price (note the integral part that the historical stock price data plays in this process). This distribution is then altered slightly, so as to ensure that the expected growth of the stock price equals that of a risk free bond. The Esscher transform is a popular technique used in order to alter the distribution in this way; this technique is discussed in Section 2.2. 
Given the probability measure obtained by estimating the objective probability measure underlying the log-returns of the stock price process, the application of the Esscher transform can be used in order to construct a probability measure, for which the price of a given option can be calculated as the discounted expected value of the payoff function of the option. This probability measure is referred to as the risk neutral measure. Section 2.3 discusses the calculation of option prices, given a risk neutral measure, using fast Fourier transforms.

Section 2.4 contains the details of a calibration procedure that is often employed in practice. This procedure ignores the pricing methodology prescribed by the abstract mathematical framework mentioned. Instead, the parameters of the model used are chosen so as to minimise some distance measure between the calculated and observed option prices. This results in a second probability measure that can be used in order to calculate option prices. In this case, the historical stock price data is not taken into account when fitting the model.

\subsection{Arbitrage free option pricing}

We assume a filtered probability space $\left(\Omega, \mathcal{F}, \mathcal{F}_{t}, \mathbb{P}\right)$, where $\mathcal{F}_{t}$ is a filtration satisfying the usual conditions; i.e. $\mathcal{F}_{t}$ is right-continuous and non-decreasing. The probability measure $\mathbb{P}$ determines the distributional properties of the log-returns of the stock price process (and therefore the properties of the stock price process itself). This measure is known as the objective probability measure.

The price $S_{t}$ of a stock (or index) at time $t$ is often modelled as a geometric process of the form

$$
S_{t}=S_{0} \exp \left(L_{t}\right),
$$

where $L_{t}$ denotes the log-return process of the stock at time $t$. Throughout this paper, all of the models used are parameterised daily. This means that $S_{t}$ denotes the stock price at the end of the $t^{\text {th }}$ business day.

The price of a European call option with strike price $K$ and time to maturity $T$ can be calculated as

$$
\pi(K, T, \mathbb{Q})=e^{-r T} \mathbb{E}^{\mathbb{Q}}\left[\max \left(S_{T}-K, 0\right)\right],
$$

where $\mathbb{Q}$ is locally equivalent to $\mathbb{P}$ and such that $e^{-r t} S_{t}$ is a $\mathbb{Q}$-martingale; for an explanation, see Chapter 6 of [4]. A measure, satisfying the two mentioned conditions is called a locally equivalent martingale measure (LEMM). It is possible that there exist multiple LEMMs. If $\mathbb{Q}$ is used in the calculation of option prices it is specifically referred to as the risk neutral measure.

Using (2) to calculate option prices entails changing probability measures from $\mathbb{P}$ to $\mathbb{Q}$. When changing measure from $\mathbb{P}$ to $\mathbb{Q}$ the probability of uncertain events changes. As a result, the processes governed by $\mathbb{P}$ and $\mathbb{Q}$ have different statistical properties. The required change of measure can be affected in a number of ways, one of which is known as the Esscher transform. 


\subsection{Esscher transform}

Let $f_{t}$ be the density of $L_{t}$ under probability measure $\mathbb{P}$. For a real number $\lambda$ such that

$$
\int_{-\infty}^{\infty} e^{\lambda x} f_{t}(x) d x<\infty
$$

define a new density to be

$$
f_{t}^{\lambda}(x)=\frac{e^{\lambda x} f_{t}(x)}{\int_{-\infty}^{\infty} e^{\lambda x} f_{t}(x) d x} .
$$

Denote by $\mathbb{P}^{\lambda}$ the probability measure under which the density of $L_{t}$ is $f_{t}^{\lambda}$.

A family of probability measures are obtained by varying the value of $\lambda$ in (3). The application of the Esscher transform entails choosing the value of $\lambda$ so that $e^{-r t} S_{t}$ forms a $\mathbb{P}^{\lambda}$-martingale (note that $\mathbb{P}^{\lambda}$ is locally equivalent to $\mathbb{P}$ by construction). For an explanation of the Esscher transform, see Chapter 6 of [15]. More technical details surrounding the Esscher transform when used in conjunction with Lèvy processes can be found in Chapter 9 of [8]. Let $\phi_{t}$ denote the characteristic function of $L_{t}$ under $\mathbb{P}$. The discounted price process $e^{-r t} S_{t}$ forms a $\mathbb{P}^{\lambda}$-martingale if, and only if,

$$
e^{r} \phi_{1}(-i \lambda)=\phi_{1}(-i(\lambda+1))
$$

see [15]. Typically, (4) has a unique solution $\lambda=\lambda^{*}$, which means $\mathbb{P}^{\lambda^{*}}$ is a LEMM, fit to be used as a risk neutral measure.

Several of the numerical results obtained in Section 4 are calculated using the Esscher transform. The application of the Esscher transform can be used to change probability measure from $\mathbb{P}$ to $\mathbb{Q}=\mathbb{P}^{\lambda^{*}}$. For each of the option pricing models considered in Section 3 , the application of the Esscher transform effects the parameters of the distribution of the increments of $L_{t}$. For each of these models, the application of the Esscher transform entails setting one of the parameters to a prespecified function of the remaining parameters. This effectively reduces the number of free parameters in each of the models by one. For a more detailed discussion of the Esscher transform, the interested reader is referred to [11].

\subsection{Option pricing using fast Fourier transforms}

The price of a European call option can be calculated directly using (2) when the distribution of $L_{t}$ is known (which is the case for each of the models considered). This can be achieved using numerical integration. However, for certain parameter sets, numerical difficulties complicate the calculation of the densities of the $N \circ I G$ and Meixner distributions; for examples as well as a proposed solution using the Fourier inversion formula, see Visagie (2018).

Carr et al. [6] show that fast Fourier transforms can be used to calculate option prices. A numerically efficient simplification to the method developed by Carr and Madan is presented in [1]. The application of this method is demonstrated below. 
In [1], Attari shows that the price of a European call option can be calculated using

$$
\begin{aligned}
\pi(K, T, \mathbb{Q})= & S_{0}-e^{-r T} K\left(\frac{1}{2}+\right. \\
& \left.\frac{1}{\pi} \int_{0}^{\infty} \frac{\left(R_{T}(\omega)+\frac{I_{T}(\omega)}{\omega}\right) \cos (\omega l)+\left(I_{T}(\omega)-\frac{R_{T}(\omega)}{\omega}\right) \sin (\omega l)}{1+\omega^{2}} d \omega\right),
\end{aligned}
$$

where $R_{T}(\omega)$ and $I_{T}(\omega)$ denote the real and imaginary parts of $\phi_{T}(\omega)$ respectively and $l=\log \left(\frac{K e^{-r T}}{S_{0}}\right)$. The formula given in (5) is used throughout the paper in order to obtain the numerical results presented.

\subsection{Calibration}

Calibrating a financial model to observed option prices entails minimising some distance measure between these prices and the option prices calculated under the risk neutral measure $\mathbb{Q}$. Consider a market with $n$ options and denote by $\pi\left(K_{j}, T_{j}, \mathbb{Q}\right)^{O, j}$ and $\pi\left(K_{j}, T_{j}, \mathbb{Q}\right)^{M, j}$ the observed price and the price under the model respectively of the $j^{t h}$ option. The root mean square error $(R M S E)$ is defined as

$$
\operatorname{RMSE}(\mathbb{Q})=\sqrt{\frac{1}{n} \sum_{j=1}^{n}\left(\pi\left(K_{j}, T_{j}, \mathbb{Q}\right)^{O, j}-\pi\left(K_{j}, T_{j}, \mathbb{Q}\right)^{M, j}\right)^{2}},
$$

where the argument $\mathbb{Q}$ is included in order to emphasize the dependence on the risk neutral measure. Throughout this paper, RMSE is chosen as the distance measure to be minimised.

Mathematically, the calibration procedure used entails choosing the risk neutral measure $\mathbb{Q}^{\star}$, where

$$
\mathbb{Q}^{\star}=\underset{\mathbb{Q} \in \mathcal{Q}}{\arg \min } R M S E(\mathbb{Q}),
$$

with $\mathcal{Q}$ the set of possible risk neutral measures. In order to guarantee the absence of arbitrage in a given market, $\mathbb{Q}$ must be a LEMM. Therefore, in order to guarantee that the market is arbitrage free, $\mathcal{Q}$ should be defined as the class of LEMMs. However, Fouque et al. [10] points out that when a calibration procedure is employed in practice, the history of $S_{t}$ is often completely ignored (or in some cases the analyst might not be able to obtain historical data on $S_{t}$ ). This means that financial practitioners often do not estimate $\mathbb{P}$ and that the requirement that the risk neutral measure be locally equivalent to the objective measure is discarded. One possible reason for the implementation of this approach is that, in some instances, the calibration procedure is substantially simplified when the local equivalence condition is ignored. This methodology is not restricted to practitioners; often when a new model is proposed in the literature the model is calibrated to option prices while ignoring the price history of the stock; see, for example, [16].

The calibration procedure used below ignores the local equivalence requirement. As a result, $\mathcal{Q}$ is the class of measures such that $e^{-r t} S_{t}$ forms a $\mathbb{Q}$-martingale for all $\mathbb{Q} \in \mathcal{Q}$. We refer to this type of calibration as full calibration. Section 4 also includes results obtained 
when using what is referred to as restricted calibration. This procedure minimises the RMSE subject to the constraint that the discrepancy between the objective and risk neutral measures not exceed a specified magnitude. As a result, restricted calibration minimises the RMSE while taking the properties of the objective probability measure into account.

\section{Option pricing models}

In this paper, the models used form part of the class of geometric Lévy models; these models are discussed below. The dynamics of the stock price process are defined in (1). We use the model obtained when the log-return process $L_{t}$ follows the $N \circ I G$ and Meixner processes. The Black-Scholes model under which $L_{t}$ is assumed to be a Brownian motion is also considered. Note that, since Brownian motion is a Lévy process, the Black-Scholes model also falls within the class of geometric Lévy process models.

The discussions of the $N \circ I G$ and Meixner distributions in Sections 3.2 and 3.3 respectively are partially based on [15].

\subsection{The Black-Scholes option pricing model}

Certainly the most famous option pricing model is the Black-Scholes model. Black and Scholes used differential equations and a replicating portfolio argument to find an option pricing formula; see [5]. In the framework of the Black-Scholes model, $L_{t}$ is assumed to be a Brownian motion, with drift $\mu \in \mathbb{R}$ and volatility parameter $\sigma>0$, under probability measure $\mathbb{P}$.

In the Black-Scholes market there exists a unique LEMM. Changing measure from $\mathbb{P}$ to the LEMM in this model entails setting the drift of the Brownian motion to

$$
\mu=r-\frac{\sigma^{2}}{2}
$$

As a result, the arbitrage free price of each option in this market is uniquely determined by the value of $\sigma$.

\subsection{The geometric $N \circ I G$ model}

The $N \circ I G$ distribution was introduced by Barndorff-Nielsen; see [2] and [3]. A random variable $X: \Omega \rightarrow \mathbb{R}$ is said to follow a $N \circ I G$ distribution with parameter set $(\alpha, \beta, \mu, \delta)$ if it has density

$$
f(x ;(\alpha, \beta, \mu, \delta))=\frac{\alpha \delta}{\pi} \exp \left(\delta \sqrt{\alpha^{2}-\beta^{2}}+\beta(x-\mu)\right) \frac{K_{1}\left(\alpha \sqrt{\delta^{2}+(x-\mu)^{2}}\right)}{\sqrt{\delta^{2}+(x-\mu)^{2}}}
$$

where $\alpha>0,|\beta|<\alpha, \mu \in \mathbb{R}, \delta>0$ and $K_{1}$ denotes the modified Bessel function of the third kind with index 1 ; see [15]. A random variable following this distribution is 
denoted by $X \sim N \circ I G(\alpha, \beta, \mu, \delta)$. The characteristic function of the $N \circ I G(\alpha, \beta, \mu, \delta)$ distribution is

$$
\phi(u ;(\alpha, \beta, \mu, \delta))=\exp \left(i \mu u-\delta\left(\sqrt{\alpha^{2}-(\beta+i u)^{2}}-\sqrt{\alpha^{2}-\beta^{2}}\right)\right) .
$$

Let $X_{j}, j=1,2, \ldots, n$, be $n$ independent and identically distributed $N \circ I G(\alpha, \beta, \mu, \delta)$ random variables, then

$$
\sum_{j=1}^{n} X_{j} \sim N \circ I G(\alpha, \beta, n \mu, n \delta) .
$$

A comment on the convolution of individual log-returns will be made in Section 4 .

If the log-return process, $L_{t}$, is a $N \circ I G$ process with parameter set $(\alpha, \beta, \mu, \delta)$, then $L_{t}$ is a Lévy process such that the random variable $L_{1}$ follows a $N \circ I G(\alpha, \beta, \mu, \delta)$ distribution. The increments of a $N \circ I G(\alpha, \beta, \mu, \delta)$ process has the distribution

$$
L_{t+s}-L_{t} \sim N \circ I G(\alpha, \beta, \mu s, \delta s),
$$

for all $s>0$.

Consider the case where $L_{t}$ follows a $N \circ I G(\alpha, \beta, \mu, \delta)$ process under $\mathbb{P}$. If the Esscher transform is used in order to find a LEMM (denoted by $\mathbb{Q}$ ), then $L_{t}$ follows a $N \circ I G\left(\alpha, \beta+\gamma^{*}, \mu, \delta\right)$ process, where $\gamma=\gamma^{*}$ is the solution to

$$
r=\mu+\delta\left(\sqrt{\alpha^{2}-(\beta+\gamma)^{2}}-\sqrt{\alpha^{2}-(\beta+\gamma+1)^{2}}\right) .
$$

This was obtained by applying (4).

In this paper, the standardised central sample moments of the historical log-returns will be compared to the standardised central moments of $L_{t}$. Denote by $m_{j}$ the $j^{\text {th }}$ standardised central moments of $L_{t}$ under the risk neutral measure used. The expected value, variance, skewness and kurtosis of $L_{t}$ are given by

$$
\begin{aligned}
m_{1}(\alpha, \beta, \mu, \delta) & =\mu t+\frac{\delta t \beta}{\sqrt{\alpha^{2}-\beta^{2}}}, \\
m_{2}(\alpha, \beta, \mu, \delta) & =\frac{\alpha^{2} \delta t}{\sqrt{\left(\alpha^{2}-\beta^{2}\right)^{3}}}, \\
m_{3}(\alpha, \beta, \mu, \delta) & =\frac{3 \beta}{\alpha \sqrt{\delta t\left(\alpha^{2}-\beta^{2}\right)^{1 / 2}}}, \\
m_{4}(\alpha, \beta, \mu, \delta) & =3\left(1+\frac{\alpha^{2}+4 \beta^{2}}{\delta t \alpha^{2} \sqrt{\alpha^{2}-\beta^{2}}}\right) .
\end{aligned}
$$

Let $M_{j}$ denote the sample equivalent of $m_{j}$ for $j=1,2,3,4$; i.e., let $M_{1}, M_{2}, M_{3}$ and $M_{4}$ respectively denote the sample mean, variance, skewness and kurtosis. In Section 4, maximum likelihood parameter estimation is used in order to fit the $N \circ I G$ distribution 
to observed log-returns. No closed-form expressions are available for the maximum likelihood parameter estimates for this distribution and, therefore, a numerical optimisation technique is used in order to arrive at the parameter estimates. However, the optimiser used requires the specification of starting values. The method of moments estimates are used for this purpose. The system of equations provided in (8) can be solved in order to arrive at the method of moments estimates given by

$$
\begin{aligned}
\hat{\alpha} & =\sqrt{\frac{3 M_{4}-4 M_{3}^{2}-9}{M_{2}\left(M_{4}-\frac{5}{3} M_{3}^{2}-3\right)^{2}}}, \\
\hat{\beta} & =\frac{M_{3}}{\sqrt{M_{2}}\left(M_{4}-\frac{5}{3} M_{3}^{2}-3\right)}, \\
\hat{\mu} & =M_{1}-\frac{3 M_{3} \sqrt{M_{2}}}{3 M_{4}-4 M_{3}^{2}-9}, \\
\hat{\delta} & =\frac{\sqrt{27 M_{2}\left(M_{4}-\frac{5}{3} M_{3}^{2}-3\right)}}{3 M_{4}-4 M_{3}^{2}-9} .
\end{aligned}
$$

\subsection{The geometric Meixner model}

A random variable $X: \Omega \rightarrow R$ is said to follow a Meixner distribution with parameter set $(\alpha, \beta, \mu, \delta)$ if it has density

$$
f(x ; \alpha, \beta, \mu, \delta)=\frac{(2 \cos (\beta / 2))^{2 \delta}}{2 \alpha \pi \Gamma(2 \delta)} \exp \left(\frac{\beta(x-\mu)}{\alpha}\right)\left|\Gamma\left(\delta+\frac{i(x-\mu)}{\alpha}\right)\right|^{2},
$$

where $\alpha>0,|\beta|<\pi,-\infty<\mu<\infty, \delta>0$ and $\Gamma(\cdot)$ denotes the complex gamma function. We denote a random variable following this distribution by $X \sim \operatorname{Meixner}(\alpha, \beta, \mu, \delta)$. The characteristic function of $X$ is

$$
\phi(u ; \alpha, \beta, \mu, \delta)=\left(\frac{\cos (\beta / 2)}{\cosh \left(\frac{\alpha u-i \beta}{2}\right)}\right)^{2 \delta} \exp (i \mu u) .
$$

Using the same notation as was used above, the first four standardised central moments of the Meixner $(\alpha, \beta, \mu, \delta)$ distribution are

$$
\begin{aligned}
& m_{1}(\alpha, \beta, \mu, \delta)=\mu+\alpha \delta \tan (\beta / 2) \\
& m_{2}(\alpha, \beta, \mu, \delta)=\frac{\alpha^{2} \delta}{2(\cos (\beta / 2))^{2}}, \\
& m_{3}(\alpha, \beta, \mu, \delta)=\sin (\beta / 2) \sqrt{2 / \delta} \\
& m_{4}(\alpha, \beta, \mu, \delta)=3+\frac{(2-\cos (\beta))}{\delta} .
\end{aligned}
$$


If the log-return process, $L_{t}$, is a Meixner process with parameter set $(\alpha, \beta, \mu, \delta)$, then $L_{t}$ is a Lévy process such that the random variable $L_{1}$ follows a Meixner $(\alpha, \beta, \mu, \delta)$ distribution. The increments of a Meixner $(\alpha, \beta, \mu, \delta)$ process are distributed as

$$
L_{t+s}-L_{t} \sim \operatorname{Meixner}(\alpha, \beta, \mu s, \delta s),
$$

for all $s>0$.

Let $L_{t}$ follow a Meixner $(\alpha, \beta, \mu, \delta)$ process under $\mathbb{P}$. In this case, if the Esscher transform is used in order to find a LEMM (denoted by $\mathbb{Q}$ ), then $L_{t}$ follows a Meixner $(\alpha, \beta+\alpha \xi, \mu, \delta)$ process, where

$$
\xi=\frac{-1}{\alpha}\left(\beta+2 \arctan \left(\frac{-\cos (\alpha / 2)+\exp ((\mu-r) / 2 \delta)}{\sin (\alpha / 2)}\right)\right) .
$$

Using the same notation for the standardised sample central moments and solving the system of equations provided in (9), one arrives at the method of moments estimates given by

$$
\begin{aligned}
\hat{\alpha} & =\sqrt{M_{2}\left(2 M_{4}-3 M_{3}^{2}-6\right)}, \\
\hat{\beta} & =\operatorname{sign}\left(M_{3}\right) \arccos \left(\frac{M_{4}-2 M_{3}^{2}-3}{M_{4}-M_{3}^{2}-3}\right), \\
\hat{\delta} & =\frac{1}{M_{4}-M_{3}^{2}-3}, \\
\hat{\mu} & =M_{1}-\hat{\alpha} \hat{\delta} \tan \left(\frac{\hat{\beta}}{2}\right) .
\end{aligned}
$$

As was the case when considering the geometric $N \circ I G$ process model, maximum likelihood estimation will be used and the Esscher transform in order to fit the geometric Meixner process model. The method of moments estimates are, once again, used as starting values for the optimisation algorithm used in order to obtain maximum likelihood estimates.

For a more detailed exposition of the Meixner distribution, the interested reader is referred to $[12]$.

\section{Empirical results}

In this section, the empirical results obtained when fitting the models described in the previous section to various sets of observed option prices are given. The financial datasets used are discussed followed by the numerical results that were obtained. The numerical results presented were obtained using Matlab. 


\subsection{Financial data used}

The options considered are obtained from the American and European financial markets. These markets were specifically selected because of their large size and number of participants. The options prices considered are those of European call options.

The first dataset considered consists of the prices of 75 options on the S\&P 500 index as at close of business on 18 April 2002. The S\&P 500 is an American capitalisation weighted stock market index based on 500 large companies. The option prices used are reported in [15]. This text also reported a risk-free interest rate in excess of dividends to be $0.7 \%$ on the date in question.

The second dataset used below consists of 144 European call option prices available on the Eurostoxx 50 index on 7 October 2003; this dataset is analysed in [16]. The Eurostoxx 50 is an index comprised of the stocks of 50 large companies from 12 countries in the Eurozone. On 7 October 2003 the index closed at 2476.61 Euros. The continuously compound risk free interest rate is assumed to be $3 \%$ per annum in accordance with the assumptions made by the authors of [16].

The final dataset discussed below contains option prices on the stock price of Google Inc. This dataset contains 545 call option prices recorded on 11 May 2012; on this date the stock price of Google Inc. closed on $\$ 605.23$. This dataset was obtained from http: //finance.yahoo.com; see [19]. In order to determine the risk-free interest rate to be used when analysing this dataset, a discount rate of six month Treasury bills in the secondary market is used. On 11 May 2012 this rate was $0.15 \%$ per annum. The dataset under consideration contained 26 prices that were either higher or lower than can be reasonably expected; for the details of the procedure used in order to identify the problematic option price values, see [17]. It is the opinion of the authors that the erroneous prices could be the result of human error (including typing mistakes) or some other shortcoming of the method used to capture the data. As a result, these prices were removed from the dataset and the analysis presented below is based on the remaining 519 option prices. Note that no values were removed from the other datasets considered.

When deciding on the historical period to consider the stock or index prices, a balance must be struck between the desire for a large dataset and the relevance of the prices. The historical period considered is used to gain information on the (constantly changing) current market conditions. If this period extends too far into the past it is possible that the market conditions have changed considerably within this historical period. In the empirical results below, historical stock and index prices for a period of one year prior to the date on which the option prices were recorded, are used. These historical prices were obtained from http://finance.yahoo.com.

\subsection{Numerical results obtained}

The numerical results presented below are obtained using the prices of the options available on the S\&P 500. Below the three different models discussed are fitted to these option prices. Each of the models is considered in turn and fitted using the various methods discussed above. As a result, a number of risk neutral measures are obtained. Given a specific risk neutral measure, the main concern is twofold. First, the accuracy of the model 
in terms of replicating the observed option prices as measured by the RMSE, needs to be determined. Secondly, the magnitude of the discrepancy between the densities associated with the estimated objective measure and the risk neutral measure respectively, needs to be determined.

For each risk neutral measure, $\mathbb{Q}$, the corresponding density function (denoted by $q$ ) is compared to a kernel density estimate obtained using the observed log-returns (this density function is denoted below by $\hat{p}$ ). This discrepancy between the measures is assessed by means of a visual comparison between the two densities, as well as by the Kullback-Leibler divergence (KLD) from $\hat{p}$ to $q$, given by

$$
D_{K L}(\hat{\mathbb{P}} \| \mathbb{Q})=\int_{-\infty}^{\infty} \hat{p}(x) \log \left(\frac{\hat{p}(x)}{q(x)}\right) d x .
$$

The first model considered is the Black-Scholes. When fitting the normal distribution to the observed log-returns and using the Esscher transform to obtain the LEMM, the parameter estimates, RMSE and KLD, are

$$
\left(\hat{\mu}, \hat{\sigma}^{2}\right)=(0,0.012), R M S E=6.553, K L D=0.020 .
$$

When using a calibration procedure that aims to minimise the RMSE between the observed and calculated option prices, the parameter estimates, RMSE and KLD are given by

$$
\left(\hat{\mu}, \hat{\sigma}^{2}\right)=(0,0.011), R M S E=6.190, K L D=0.024 .
$$

These results shown in (10) and (11) differ very little. Due to the similarity in the risk neutral measures used (as is demonstrated by the small value of the KLD), the option prices calculated under the two risk neutral measures are similar.

Figure 1 shows a kernel density estimate of the distribution of the log-returns as well as the two risk neutral densities associated with the estimation procedure (denoted by "Estimated density") and the calibration procedure (denoted by "Pricing density") respectively. The figure indicates that the three densities in question are similar.

Figure 2 shows the observed market prices as well as the calculated prices where the risk neutral density is obtained using the results of the calibration procedure. The market prices of the options are shown using black lines; the lines are obtained using linear interpolation between the option prices considered. The same convention is used in Figures 3 and 4. The calculated prices fit the market prices, as a function of time to maturity $(T)$ and moneyness $\left(\log \frac{K}{S_{0}}\right)$ quite well for at-the-money (ATM) options, where $K=S_{0}$, as well as for some of the in-the-money (ITM) options, where $K<S_{0}$. However, for all of the various values of $T$, most of the out-the-money (OTM) calibrated option prices are higher than the observed market prices. In Figures 2, 3 and 4, different colours are used in order to distinguish between different times to maturity.

Attention is now turned to the results obtained using the geometric $N \circ I G$ model. For this model, no closed-form formulae are available for the maximum likelihood estimators for the parameters. In order to perform maximum likelihood estimation, Nelder-Mead optimisation (see [13]) is applied using Matlab's fminsearch. This method requires the 


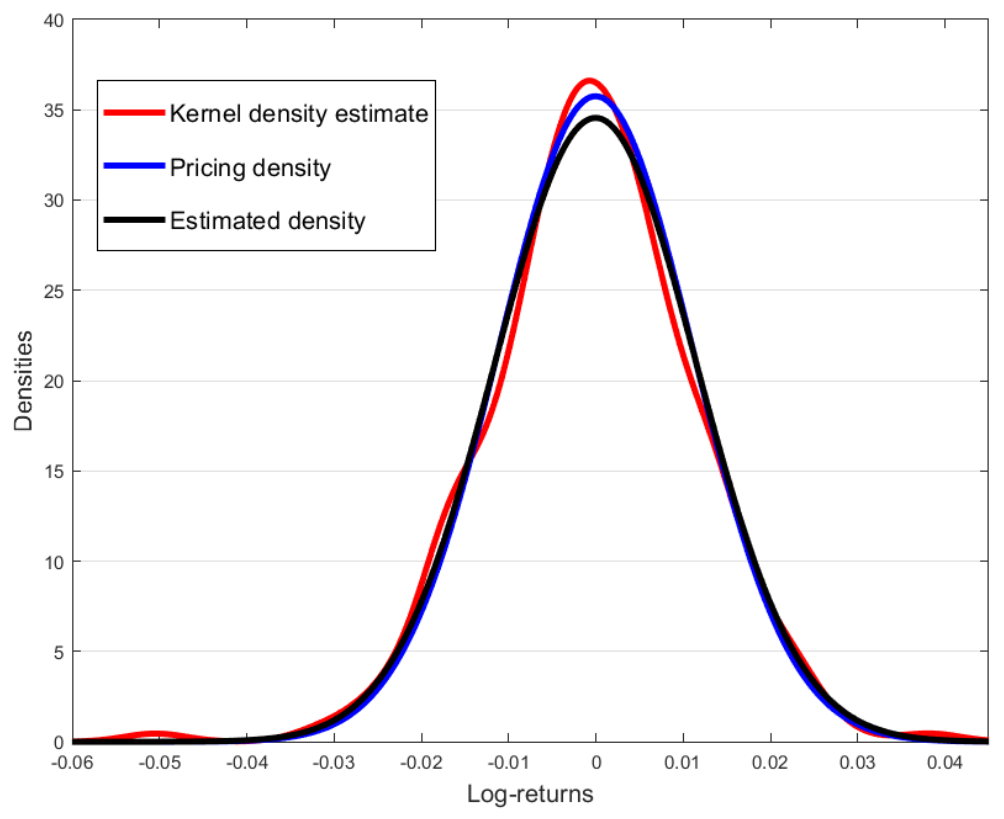

Figure 1: Density functions for the Black-Scholes model.

specification of starting values. The method of moments estimators (for which the formulae are provided in Section 3) are used as starting value for the optimisation algorithm.

When performing parameter estimation using maximum likelihood estimation, and using the Esscher transform to obtain a LEMM, the results obtained are

$$
(\hat{\alpha}, \hat{\beta}, \hat{\mu}, \hat{\delta})=(168.396,-9.203,0.001,0.022), R M S E=6.442, K L D=0.011 .
$$

When comparing the results reported in (12) to those reported in (10), it is clear that the additional flexibility that the $N \circ I G$ distribution offers over that of the normal distribution reduces the KLD. However, this improved ability to model the shape of the density function does not translate into a substantial reduction in the RMSE.

Next, the calibration of the geometric $N \circ I G$ model to the observed stock prices is considered. Since no closed-form expressions are available for the parameter values to be used, Nelder-Mead optimisation is applied a second time. In order to obtain starting values for the optimisation algorithm, a random procedure is used as follows. A range of possible starting values for each of the parameters is specified; these ranges are chosen so as to include parameter values that are deemed likely to be close to the values providing an optimal calibration (since the optimisation problem considered is generally non-convex and the optimiser used may converge to a local optimum). For each of the parameters, one thousand random starting values are generated from a uniform distribution on the chosen range. The RMSE is calculated for each of the one thousand resulting parameter sets, and the parameter set with the smallest RMSE is used as the starting values for the optimisation algorithm. The individual ranges used are omitted for the sake of brevity, but can be obtained from the authors upon request. 


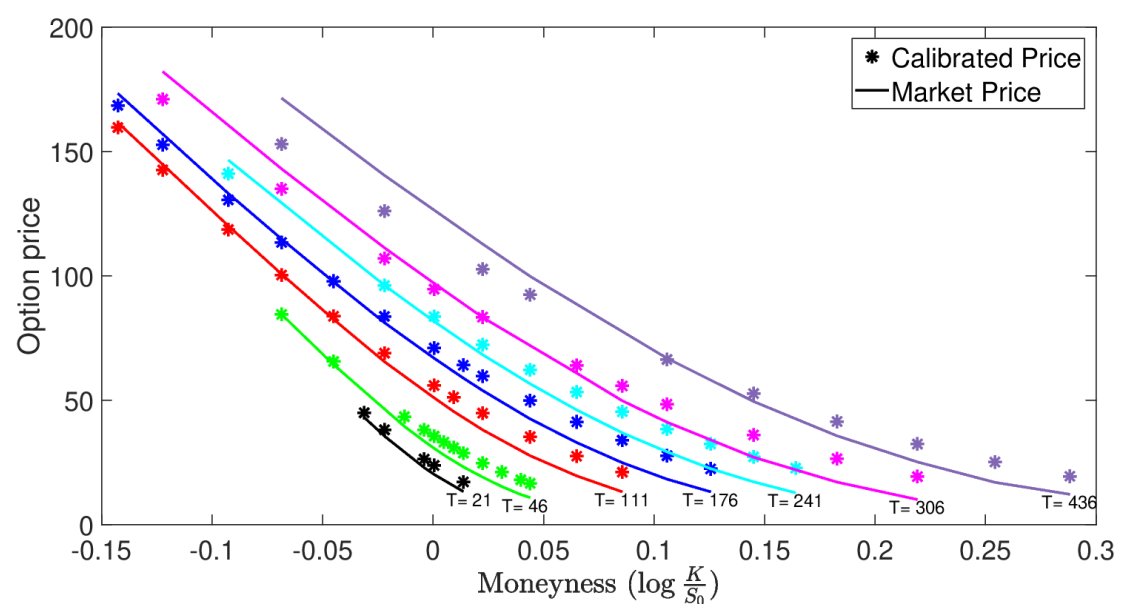

Figure 2: Market prices and calibrated option prices under the Black-Scholes model.

This paper numerically demonstrates that, in certain cases, there is an appreciable difference between the densities associated with the risk neutral and estimated objective densities. Note that, in the case of the geometric $N \circ I G$ model, both of these measures are obtained using an optimisation procedure, but the starting values used by the optimiser differs. The authors would like to emphasise that the observed differences in the densities is not explained by the difference in the starting values used. The vast differences in the densities obtained remain if the same starting values are used in the two optimisation procedures.

When using a calibration procedure in order to fit the geometric $N \circ I G$ model to the observed option price data, the results obtained are given by

$$
(\hat{\alpha}, \hat{\beta}, \hat{\mu}, \hat{\delta})=(8.550,-5.334,0.001,0.001), \quad R M S E=3.027, K L D=1.299
$$

The value of the RMSE reported in (13) is noticeably smaller than the corresponding value reported in (11). Figure 3 shows the observed market prices as well as the calculated prices where the risk neutral density is obtained using the results of the calibration procedure. In this case, the results for ITM option prices are similar to those obtained using the BlackScholes model. However, the calibrated options prices for OTM options corresponds more closely to the observed market prices. This observation is confirmed in Figure 4, in which the calculated prices using the calibrated Black-Scholes and geometric $N \circ I G$ models are compared. The results are shown for ATM as well as OTM options.

We conclude that, for this specific set of option prices, the risk neutral measure of the $N \circ I G$ model can be chosen such that the calculated prices of the options correspond more closely to the observed values than is the case when using the Black-Scholes model. The results reported in (12) also indicate that the risk neutral measure can be chosen in such a way that the corresponding density closely matches that of the observed logreturns. However, the densities associated with these two risk neutral measures (one obtained using maximum likelihood parameter estimation and the other obtained using calibration) differ substantially; note that the KLD reported for the calibration related 


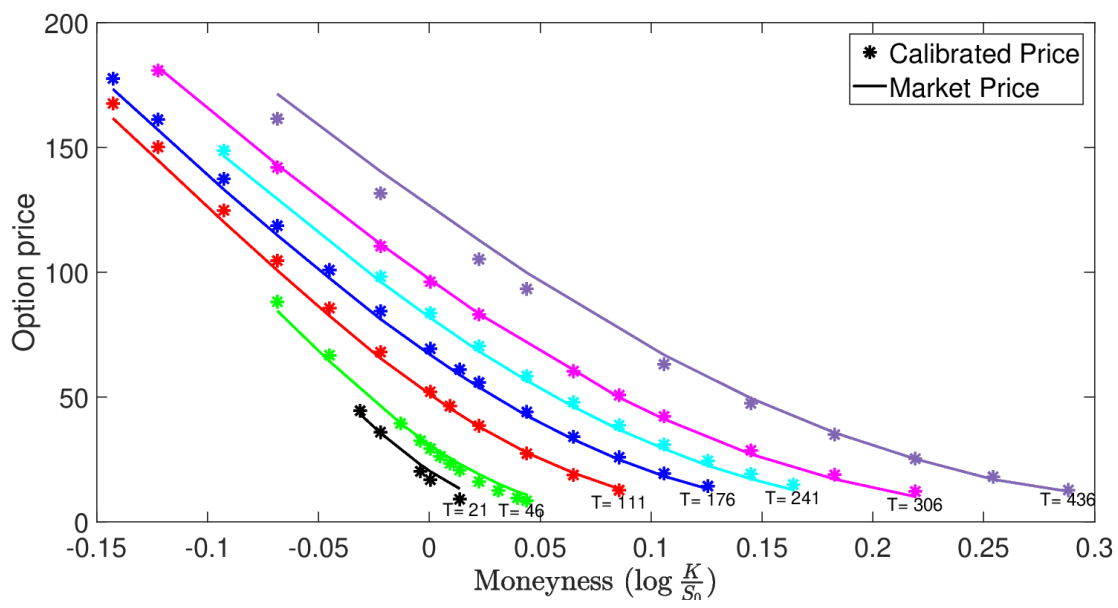

Figure 3: $\quad$ Market prices and calibrated option prices under the geometric $N \circ I G$ model.

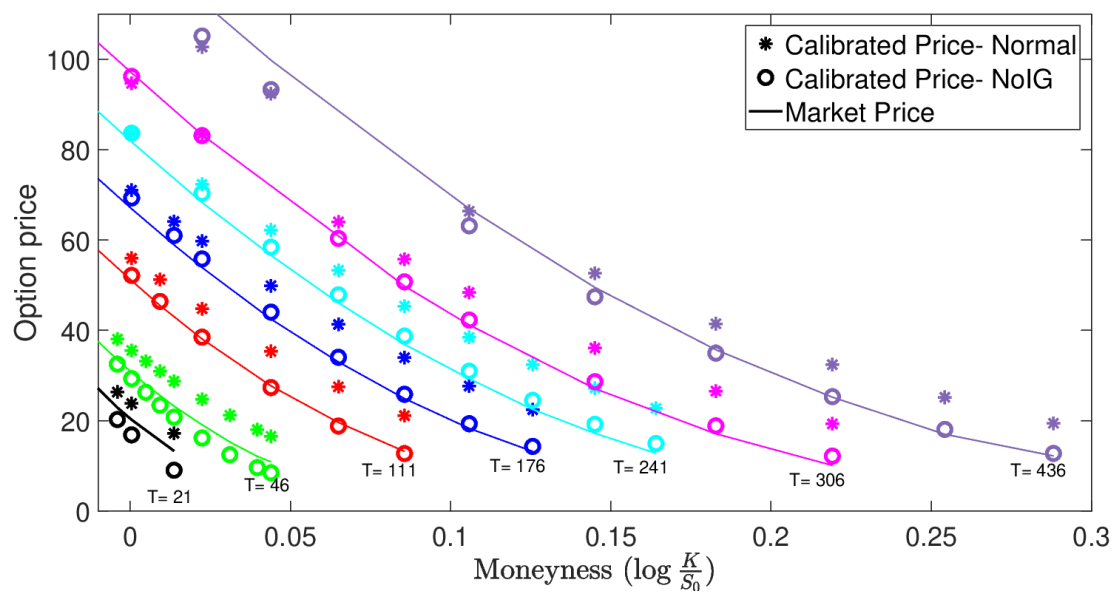

Figure 4: Market option prices compared to the calibrated option prices under the Black-Scholes and geometric $N \circ I G$ models.

to the geometric $N \circ I G$ model is appreciably larger than the other KLDs reported thus far. In order to further appreciate this discrepancy, consider Figure 5. This figure shows a kernel density estimate of the observed log-returns using a solid line with the risk neutral density obtained by calibration using a dashed line. It is clear that there is a substantial difference between the two densities.

As was discussed above, it is to be expected that the density obtained using a calibration procedure would differ from the estimated density based on historical log-returns. However, the magnitude of the observed difference when calibrating the geometric $N \circ I G$ model to the observed option prices is unexpected. Consider, for example, the third and fourth standardised central moments associated with the risk neutral measure; the skewness is calculated to be -26.3 while the kurtosis is 1516 . The corresponding sample quantities based on the observed log-returns data are -0.21 and 4.17 respectively. While it is entirely 


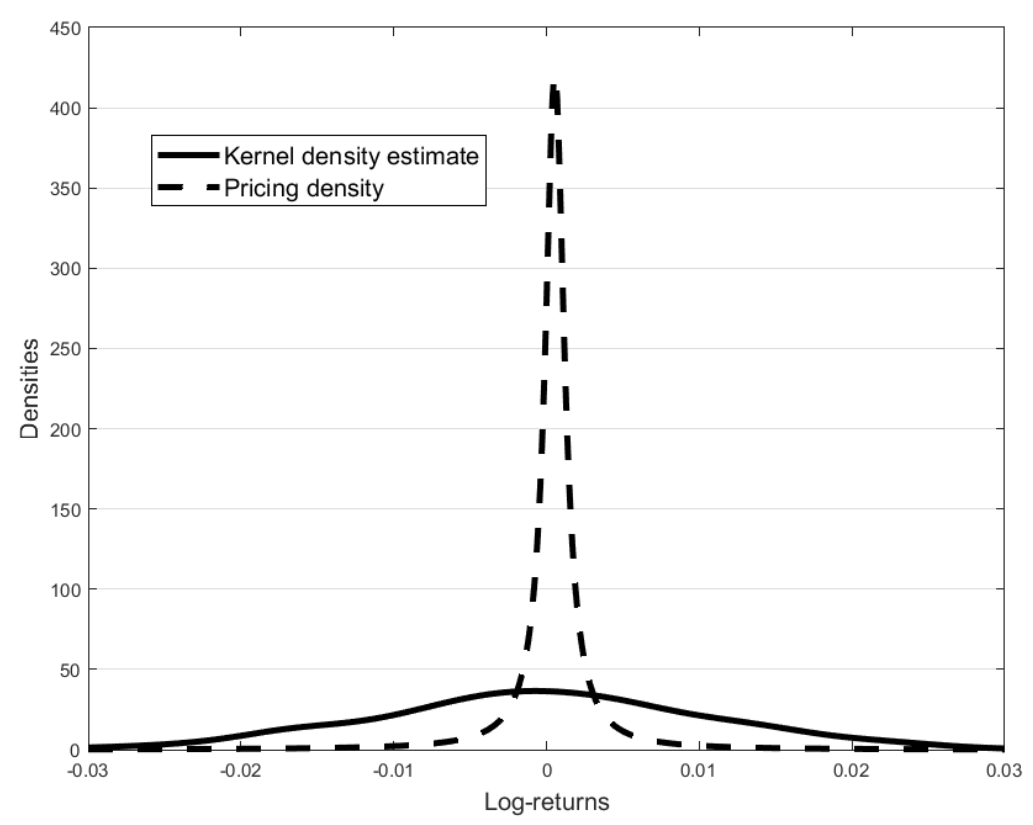

Figure 5: Density functions for the geometric $N \circ I G$ model (full calibration).

possible for the moments associated with the risk neutral measure to be different from those associated with the observed log-returns, it seems unlikely that the future skewness of the distribution will be 123 time that of observed historical skewness. When comparing the kurtosis, this difference is even more pronounced. In this case the ratio between the kurtosis of the risk neutral measure and the historical log-retuns is calculated to be 364 .

The large difference between the objective and risk neutral measures means that the prices of the options are calculated under the assumption that the future behaviour of the logreturns will differ drastically from its previous behaviour. As a result, if the risk neutral measure provides an accurate description of the future distribution of the log-returns, then the density of the log-returns observed after 18 April 2002 should resemble that of the risk neutral measure and not the historical density. Figure 6 shows two kernel density estimates associated with log-returns for one year before and one year after the mentioned date. Although there is a noticable difference between the two estimated densities, the difference is much less pronounced than the difference between the density estimate based on the historical log-returns and the risk neutral measure obtained using calibration.

The discrepancy between the historical density and the future density can be measured using the KLD. Consider the two kernel density estimates shown in Figure 6. The KLD between the historical and future densities is calculated to be 0.14 , which is only a small fraction of the reported KLD in (13). In fact, if the KLD in a similar manner on two year rolling windows starting on 1 January 1990 and ending on 5 April 2019, an observation is made that $95 \%$ of the calculated KLD values are in the interval $[0.0163 ; 0.4364]$. 


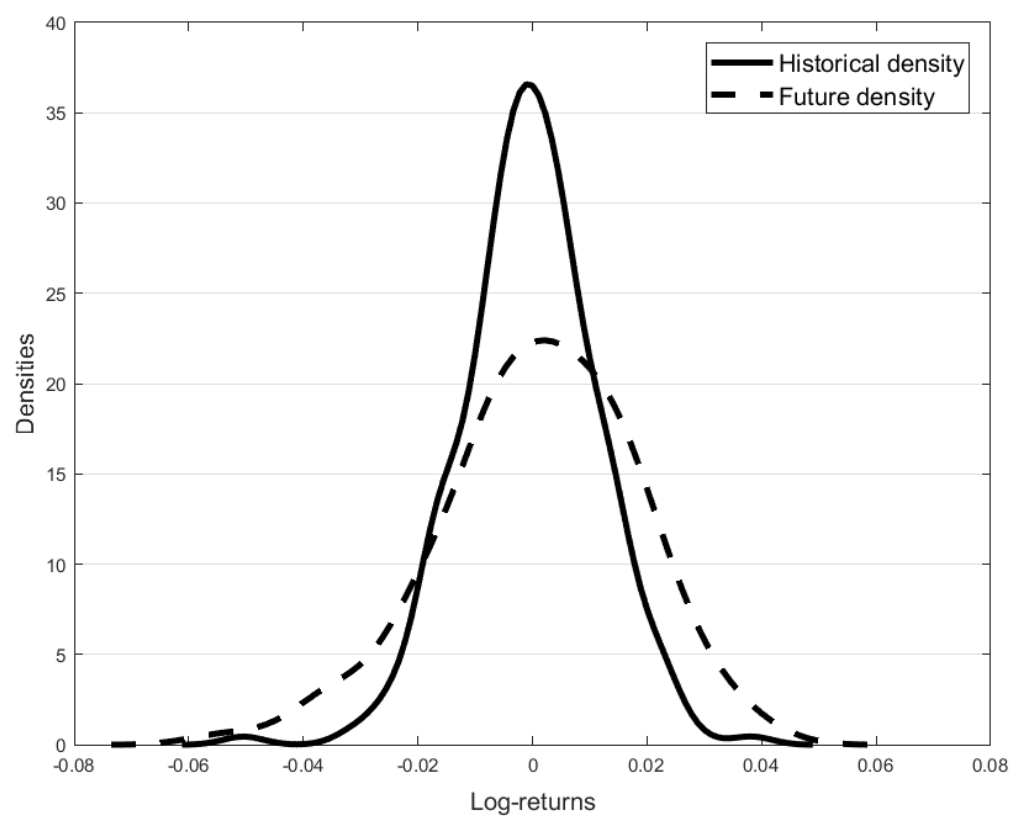

Figure 6: Historical and observed future density functions.

The magnitude of the difference between the estimated objective measure and the risk neutral measure obtained using calibration is disconcerting. This large deviation is neither observed when considering the distribution of future returns, nor is there a theoretical justification for this difference. However, the calibration procedure is clearly able to reduce the RMSE. In an attempt to find a compromise between a small RMSE value and a risk neutral density that closely resembles the estimated density of the historical log-returns, attention is turned to a procedure that shall be referred to as restricted calibration.

The calibration procedure used above allows the parameters of the model to vary freely in an attempt to minimise the RMSE. The only restriction imposed is that the discounted stock price process be a martingale under the resulting probability measure. When considering a restricted calibration, a second restriction is imposed on the parameters of the model. This restriction is that the KLD between the estimated objective measure and the resulting risk neutral measure does not exceed a specified value, say $d$. Mathematically, the restricted calibration procedure can be expressed using (6), where $\mathcal{Q}$ is defined to be the collection of all measures $\mathbb{Q}$ such that $e^{-r t} S_{t}$ forms a martingale under $\mathbb{Q}$ and $D_{K L}(\hat{\mathbb{P}} \| \mathbb{Q}) \leq d$. When using the restricted calibration procedure with a conservative $d=0.5$, for example, the results obtained for the geometric $N \circ I G$ model are

$$
(\alpha, \beta, \mu, \delta)=(27.660,-14.083,0.001,0.002), \quad R M S E=4.810, \quad K L D=0.5 .
$$

These results were obtained using the parameters in (12) as starting values. Interestingly, the KLD for this probability measure is calculated to be exactly 0.5 , meaning that the restricted calibration procedure converged to a risk neutral measure as different from the objective probability measure (as measured by the KLD) as was allowed. Figure 7 shows 
the kernel density estimate of historical log-returns as well as the risk neutral density obtained using restricted calibration. Although there is a clear difference between the estimated objective and risk neutral measures, this difference is much less pronounced than is the case when using full calibration.

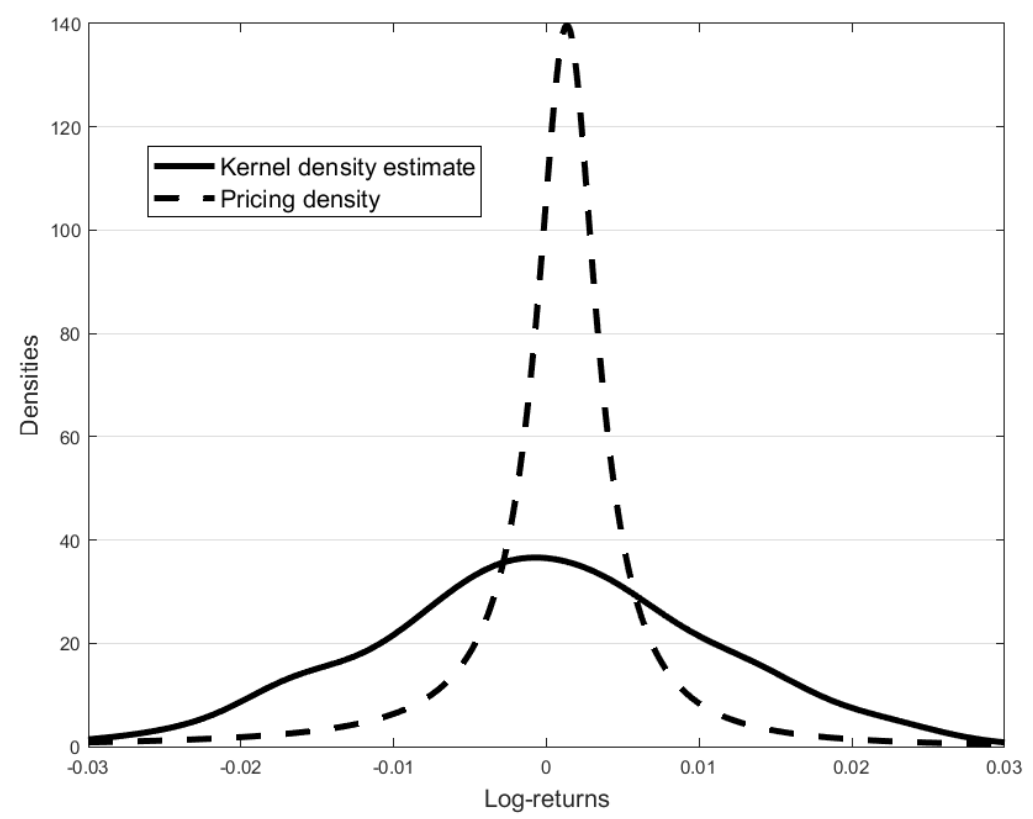

Figure 7: Density functions for the geometric $N \circ I G$ model (restricted calibration).

When using restricted calibration, the value used for $d$ is subjective and can be chosen by the modeller. The results discussed above seem to indicate that the RMSE can be decreased at the price of allowing greater differences between the estimated objective and risk neutral measures (as measured by the KLD). One possible way of performing calibration is to vary the value of $d$ when using restricted calibration and to choose a value of $d$ that allows an acceptable trade off between the RMSE and KLD measures. In order to illustrate this, a range of $d$ values was selected and the corresponding RMSE was calculated in each case. The results are shown in Figure 8. The figure clearly indicates that the RMSE is a decreasing function of the maximum KLD value allowed.

We conclude the discussion about the geometric $N \circ I G$ distribution with a note about the fact that the model under discussion is parameterised daily. The excessive differences between the estimated objective and risk neutral densities obtained using full and restricted calibration can be seen in Figures 5 and 7. In the majority of scientific papers, the models used are parameterised annually (see, for example, [16]), meaning that the reported parameters correspond to the density of the annual log-returns. However, these parameters are not estimated based on annual log-returns, but rather daily log-returns. Figure 7 shows the densities associated with the annual log-returns for each of the three risk neutral measures calculated for the geometric $N \circ I G$ model. When comparing the densities based on a annual time scale (using the convolution formula provided in (7)), 


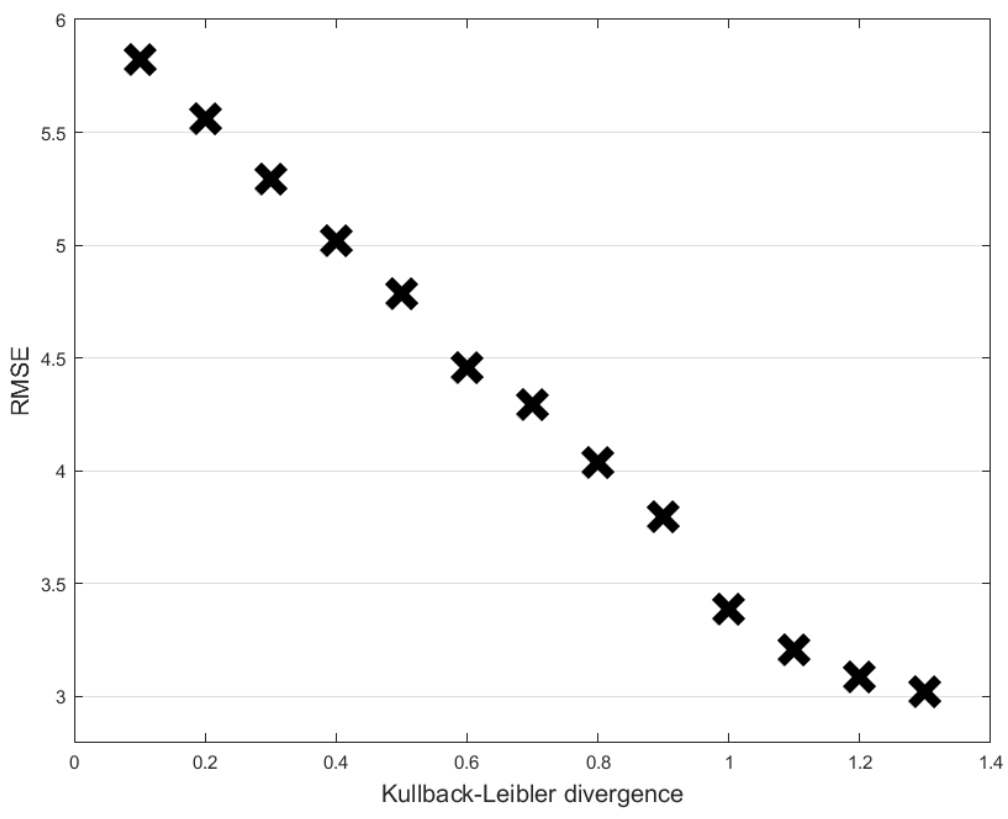

Figure 8: RMSE as a function of the KLD.

the observed differences between the density functions is perceived to be markedly less pronounced. The authors believe that this mismatch between the time scales associated with the data used and the reported parameters is misleading and should be avoided.

In order to demonstrate that the results discussed above are not limited to the geometric $N \circ I G$ model, attention is now turned to the geometric Meixner model. When fitting the Meixner distribution to the observed historical log-returns and applying the Esscher transform,

$$
(\hat{\alpha}, \hat{\beta}, \hat{\mu}, \hat{\delta})=(0.014,-0.120,0.001,1.294), \quad R M S E=6.448, \quad K L D=0.011 .
$$

Note the similarity between the RMSE obtained using the geometric $N \circ I G$ and Meixner models. When using a full calibration procedure in order to obtain a risk neutral measure,

$$
(\alpha, \beta, \mu, \delta)=(0.021,-2.929,0.001,0.008), \quad R M S E=3.733, \quad K L D=2.921
$$

is obtained. Again, the RMSE obtained using this model is similar to the RMSE obtained using the previous model. Figure 10 compares the kernel density estimate obtained using the observed historical log-returns to the density of the risk neutral measure obtained using full calibration. In this case the KLD is even more extreme than was observed when using the geometric $N \circ I G$ model.

As was the case before, the risk neutral measure obtained using a full calibration procedure clearly differs substantially from the estimated objective probability measure. As a result, we consider the restricted calibration procedure where the KLD between the estimated objective and risk neutral densities is not allowed to exceed 0.5. Using this calibration 


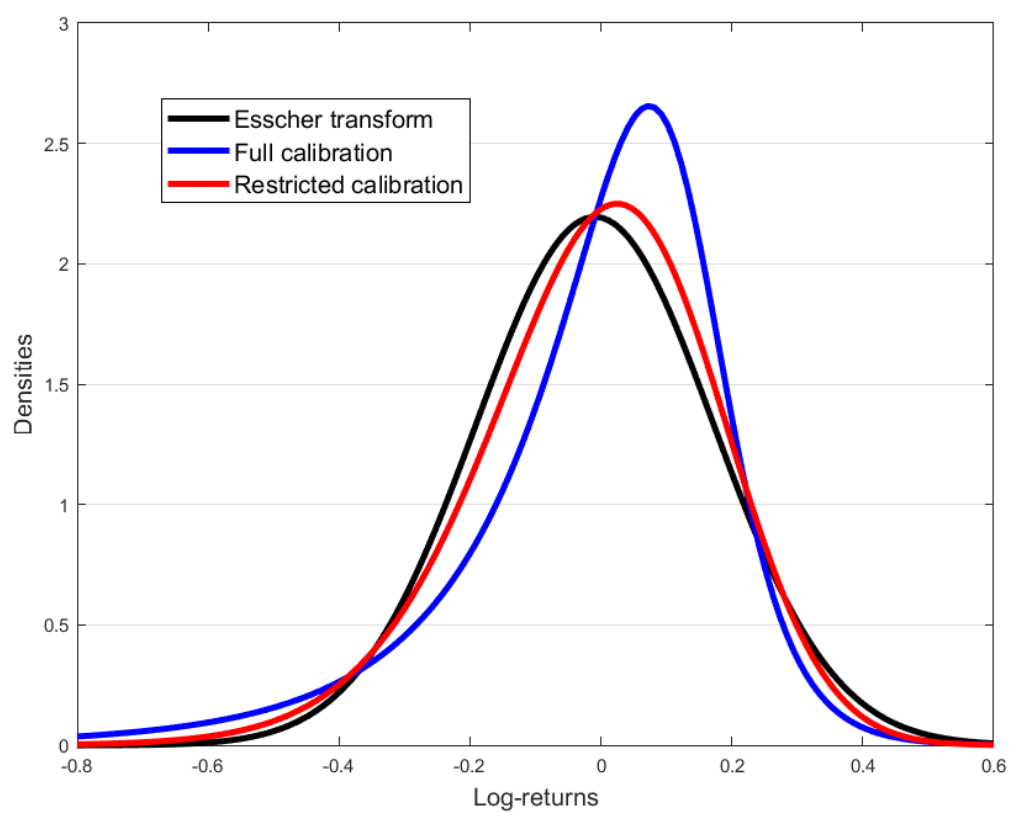

Figure 9: Annual risk neutral densities for the geometric $N \circ I G$ model.

procedure,

$$
(\alpha, \beta, \mu, \delta)=(0.030,-2.001,0.004,0.085), \quad R M S E=5.313, \quad K L D=0.5
$$

is obtained. Figure 11 compares the estimated objective density as well as the risk neutral measure obtained using restricted calibration as before.

Note the similarities between the results obtained when using the geometric $N \circ I G$ and the geometric Meixner models. These similarities are observed in the case where the maximum likelihood estimation and the Esscher transform are employed as well as the cases where full and restricted calibration are used. For ease of reference, Table 1 provides a summary of the results obtained using each of the models and fitting procedures. The following short-hand notation is used in the tables below; ET is short for Esscher transform (this indicates that the results were obtained using maximum likelihood estimation and the Esscher transform), FC is short for full calibration, while RC is short for restricted calibration.

Tables 2 and 3 contain the results associated with the Eurostoxx and Google Inc. data respectively.

When comparing the results presented in Tables 2 and 3 above to those in Table 1, several similarities are noticed. When using maximum likelihood estimation and the Esscher transform, the risk neutral measures obtained under the geometric $N \circ I G$ and Meixner models deviates from the estimated objective probability appreciably less than is the case under the Black-Scholes model. However, when using these probability measures to calculate arbitrage free option prices, there is no corresponding reduction in the RMSE 


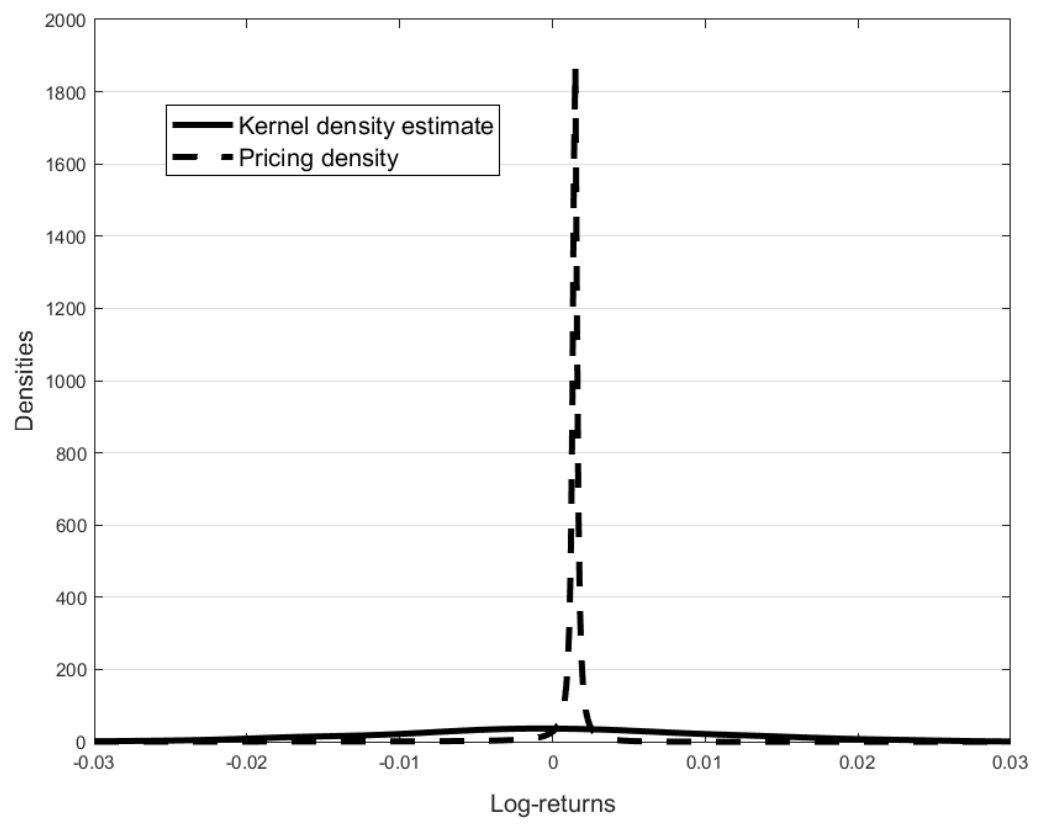

Figure 10: Density functions for the geometric Meixner model (full calibration).

\begin{tabular}{llll}
\hline Models & Parameters & RMSE & K $\boldsymbol{L} \boldsymbol{D}$ \\
\hline Black-Scholes (ET) & $(0,0.012)$ & 6.553 & 0.020 \\
Black-Scholes (C) & $(0,0.011)$ & 6.190 & 0.024 \\
$N \circ I G(\mathrm{ET})$ & $(168.396,-9.203,0.001,0.022)$ & 6.442 & 0.011 \\
$N \circ I G(\mathrm{FC})$ & $(8.550,-5.334,0.001,0.001)$ & 3.027 & 1.299 \\
$N \circ I G(\mathrm{RC})$ & $(27.660,-14.083,0.001,0.002)$ & 4.810 & 0.500 \\
Meixner (ET) & $(0.014,-0.120,0.001,1.294)$ & 6.448 & 0.011 \\
Meixner (FC) & $(0.021,-2.929,0.001,0.008)$ & 3.733 & 2.921 \\
Meixner (RC) & $(0.030,-2.001,0.004,0.085)$ & 5.313 & 0.500 \\
\hline
\end{tabular}

Table 1: Results for the S\&P 500 option prices.

observed when changing from the Black-Scholes to either of the other models. When the results obtained using calibration are considered, it is noticed that, in this case, the geometric $N \circ I G$ and Meixner process models far outperform the Black-Scholes in terms of the RMSEs obtained. However, the discrepancy between the risk neutral measure used and the estimated objective measure (as measured by the KLD) is substantial. In each of the cases considered, the geometric $N \circ I G$ process model slightly outperforms the geometric Meixner model in terms of the calculated RMSEs; the former model also generally provides smaller KLDs than is the case for the latter model.

Note that the three numerical examples used are based on data recorded at three different times (although two of the three dates in question differ only by roughly one and a half year). Also, the three datasets used were recorded in two different financial markets; the American and European markets. Finally, the examples used consider options for which the underlying assets comprise both a single stock and two indices. Of course, no general 


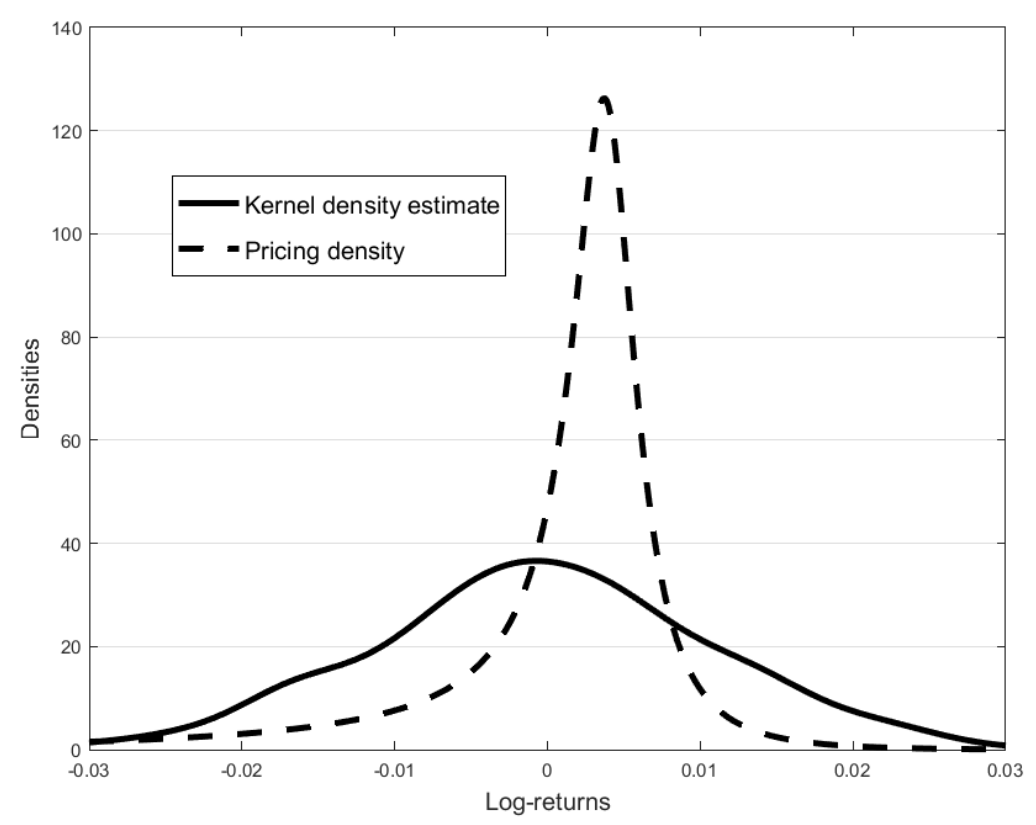

Figure 11: Density functions for the geometric Meixner model (restricted calibration).

\begin{tabular}{llll}
\hline Models & Parameters & RMSE & K LD \\
\hline Black-Scholes (ET) & $(0,0.020)$ & 131.659 & 0.020 \\
Black-Scholes (C) & $(0,0.014)$ & 31.267 & 0.208 \\
$N \circ I G(\mathrm{ET})$ & $(74.873,9.873,-0.004,0.030)$ & 132.667 & 0.009 \\
$N \circ I G(\mathrm{FC})$ & $(7.312,-5.472,0.001,0.001)$ & 13.180 & 1.880 \\
$N \circ I G(\mathrm{RC})$ & $(37.217,-19.740,0.003,0.005)$ & 27.893 & 0.500 \\
Meixner (ET) & $(0.032,0.312,-0.004,0.792)$ & 132.164 & 0.008 \\
Meixner (FC) & $(0.003,-3.127,0.001,0.003)$ & 14.254 & 17.561 \\
Meixner (RC) & $(0.002,-2.391,0.059,9.491)$ & 30.889 & 0.497 \\
\hline
\end{tabular}

Table 2: Results for the Eurostoxx option prices.

conclusions can be drawn based on the examples included. However, the variety mentioned above seems to suggest that the observations made are not limited to a single time, market or type of underlying asset.

\section{Conclusion}

In this paper, three option pricing models are considered; the Black-Scholes model as well as the geometric normal inverse Gaussian $(N \circ I G)$ and Meixner models. Under each of these models, the log-return process is modelled using a Lévy process. Under the BlackScholes model, the log-returns follow a normal distribution. Under the remaining models, the log-returns are realised from more flexible models. 


\begin{tabular}{llll}
\hline Models & Parameters & RMSE & K $\boldsymbol{D} \boldsymbol{D}$ \\
\hline Black-Scholes (ET) & $(0,0.019)$ & 10.141 & 0.060 \\
Black-Scholes (C) & $(0,0.018)$ & 10.095 & 0.058 \\
$N \circ I G(\mathrm{ET})$ & $(47.516,-7.689,0.002,0.015)$ & 10.028 & 0.013 \\
$N \circ I G(\mathrm{FC})$ & $(6.729,-5.527,0.001,0.001)$ & 8.106 & 1.370 \\
$N \circ I G(\mathrm{RC})$ & $(18.187,-12.250,0.003,0.003)$ & 9.104 & 0.500 \\
Meixner (ET) & $(0.047,-0.382,0.003,0.292)$ & 10.037 & 0.014 \\
Meixner (FC) & $(0.002,-3.136,0.002,0.003)$ & 8.570 & 24.266 \\
Meixner (RC) & $(0.065,-2.083,0.005,0.044)$ & 9.475 & 0.500 \\
\hline
\end{tabular}

Table 3: Results for the Google Inc. option prices.

Several methods can be used in order to fit a given financial model to observed data. A method often employed in practice is a calibration technique that entails choosing the parameters of the model so as to minimise some discrepancy measure between the observed and calculated option prices. This paper provides a numerical comparison between the density of the log-returns obtained using calibration and the estimated density. The paper shows that, in the examples considered, the geometric $N \circ I G$ and Meixner models outperform the Black-Scholes model in terms of their ability to provide option prices that resemble those observed in the market. However, when using these models, the densities resulting from the calibration procedure are substantially different from the estimated density of the observed log-returns. This means that the calculation of the option prices are performed under materially different assumptions than are observed in the market. This phenomenon is not observed when using the Black-Scholes model.

The aim of this paper is not to discourage the use of flexible financial models like the geometric $N \circ I G$ and Meixner models. In fact, in the examples considered, these models are able to outperform the Black-Scholes model in terms of the root mean square error (RMSE) calculated between the observed option prices and the option prices calculated under the various models. The results presented in the paper serve to emphasise that, when a calibration procedure is used in order to fit flexible models containing multiple parameters, the resulting density function will not necessarily resemble that of the logreturns observed in the market. A possible solution to this problem is proposed; the calibration might be restricted in the sense that the parameters of the model are chosen so as to minimise the RMSE between the observed and calculated option prices, subject to the restriction that the density obtained using calibration does not differ more than a prespecified amount from the estimated density of the observed log-returns. Generally, the enforcement of this restriction will result in larger RMSE values. However, the use of this method ensures that the density obtained using calibration resembles the estimated density of the log-returns.

\section{References}

[1] Attari M, 2004, Option pricing using Fourier transforms: a numerically efficient simplification., Working paper.

[2] Barndorf-Nielsen OE, 1997, Normal inverse Gaussian distributions and stochastic volatility modelling, Scandinavian Journal of Statistics, 24, pp. 1-13. 
[3] Barndorf-Nielsen OE, 1998, Processes of normal inverse Gaussian type, Finance and Stochastics, 2, pp. $41-68$.

[4] Barndorf-Nielsen OE \& Shiryaev A, 2010, Change of time and change of measure, World Scientific Publishing Co. Pte. Ltd., Singapore.

[5] Black F \& Scholes M, 1973, The pricing of options and corporate liabilities, The Journal of Political Economy, 3, pp. 637-654.

[6] CARR P \& MADAN DB, 1999, Option valuation using the fast Fourier transform., Journal Computational Finance, 4, pp. 61-73.

[7] Cont R, 2001, Empirical properties of asset returns: stylized facts and statistical issues, Quantitative Finance, 1, pp. 223-236.

[8] Cont R \& Tankov P, 2004, Financial modelling with jump processes, Chapman and Hall/CRC., London, United Kingdom.

[9] FAMA EF, 1970, Efficient capital markets: a review of theory and empirical work, The Journal of Finance, 25, pp. 383-417.

[10] Fouque JP, Papanicolaou G \& Sircar KR, 2001. Derivatives in financial markets with stochastic volatility, Cambridge University Press, Cambridge, United Kingdom.

[11] Gerber HU \& ShiU ESW, 1994, Option pricing by Esscher transforms (with discussions), Transactions of the Society of Actuaries, 46, pp. 99-191.

[12] Grigelionis B, 1999, Processes of Meixner type, Lithuanian Mathematical Journal, 39, pp. 33-41.

[13] Nelder JA \& MeAd R, 1965, A simplex method for function minimization, The Computer Journal, 7, pp. $308-313$.

[14] Ross S, 2015, The recovery theorem, The Journal of Finance, 70, pp. 615-647.

[15] Schoutens W, 2003, Lévy Processes In Finance: Pricing Financial Derivatives, John Wiley \& Sons Ltd, Chichester, England.

[16] Schoutens W, Simons E \& Tistaert J, 2004, A perfect calibration! Now what?, Wilmott magazine, 8, pp. 66-78.

[17] Visagie IJH, 2015, On the calibration of Lévy option pricing models, PhD thesis, North-West University.

[18] ViSAGIE IJH, 2018, On parameter estimation in multi-parameter distributions, Statistics, Optimization and Information Computing, 6, pp. 452-467.

[19] Yahoo! Finance, Google Inc. (GOOG), [Online], [Cited May 12 ${ }^{\text {th }}$, 2012], Available from http: //finance $\cdot$ yahoo $. \mathrm{com} / \mathrm{q} / \mathrm{pr}$ ? $\mathrm{s}=\mathrm{GOOG}$. 